\title{
A Freeze Drying Sample Preparation Method for Correlative Light and Scanning/Transmission Electron Microscopy
}

\author{
Kunihiro Uryu $^{1}$, Nadine Soplop ${ }^{1}$, Devrim Acehan ${ }^{1}$, Charles M. Rice ${ }^{2}$, Maria Teresa Catanese ${ }^{2,3}$, Ayuko \\ Hoshino $^{4}$, David Lyden ${ }^{4}$ \\ ${ }^{1 .}$ Electron Microscopy Resource Center, The Rockefeller University, New York, NY, USA. \\ 2. Center for the Study of Hepatitis C, Laboratory of Virology and Infectious Disease, The Rockefeller \\ University, New York, New York, USA \\ 3. Department of Infectious Diseases, King's College London, London, United Kingdom \\ 4. Children's Cancer and Blood Foundation Laboratories, Departments of Pediatrics, and Cell and \\ Developmental Biology, Drukier Institute for Children's Health, Meyer Cancer Center, Weill Cornell \\ Medicine, New York, New York, USA.
}

Electron microscopy (EM) provides detailed structural information of biological samples but limited functional insight. In contrast, light microscopy enables dynamic imaging of fluorescently labeled molecule and organelles but misses the underlying structures. Correlative Light and Electron Microscopy (CLEM) combines the strengths of these two approaches by allowing the precise localization of fluorescent molecules in the context of the surrounding ultrastructure of a biological sample of interest (i.e., cell, tissue, organism). In doing so, CLEM provides a powerful tool for revealing mechanistic links between the position and putative functions of the molecules that are being tracked.

In recent years, new CLEM methodologies have been developed to overcome remaining technological challenges, namely a sample preparation protocol that works across multiple imaging systems including light microscopy, scanning EM (SEM) or transmission EM (TE(TEM), , while preserving both the native structural organization of cells/tissues and fluorescence. An important unmet need is the maintenance of native conformations of lipid-rich structures, such as cell membranes, which are prone to deformation by the organic solvents that are used in EM. The other challenge lies in dealing with fluorescent molecules that are easily bleached by organic solvents. Given these challenges, we tested a series of methods, aimed to achieve the following goals: 1) Retain the integrity of lipid-rich membranes; 2) Resolve structures to a nanometer resolution; 3) Preserve fluorescent signals; and 4) Apply CLEM to a broader range of biological samples. We devised a three-step-procedure three-step procedure: 1) Plunge freezing for instantaneous fixation; 2) Freeze drying in cold nitrogen gas $\left(\mathrm{GN}_{2}\right)$; and 3) Critical point drying (CPD). Here, we report a new sample preparation procedure, coupled with a freeze drying method that relies on gaseous nitrogen (FDGN 2 method), which results in unprecedented structural preservation of biological samples using high vacuum SEM${ }^{\mathbf{1}}$.

We have also extended the applicability of the $\mathrm{FDGN}_{2}$ method to TEM. After dehydration (and possibly SEM examination), samples were infiltrated with Lowicryl HM20 resin and embedded at $-50^{\circ} \mathrm{C}$. Thin sections were cut and examined by fluorescence microscopy and TEM. Figure 1 demonstrates CLEM examples. A fluorescent image of exogenous exosomes in a fibroblast, labeled by FMI-43 (Green)/PKH26 (red), is overlaid with a topographic view of the cell (a). A thin TEM section of a fibroblast with exogenous exosomes labeled by a green fluorescence dye (FM1-43) was examined fluorescence signal and ultrastructure (b). A hepatoma cell with RFP tagged mitochondrially-tethered IFN- $\beta$ promoter stimulator protein was examined by an epi-fluorescent microscope subsequently subjected for TEM thin sectioning for ultrastructure analysis (c). These results provide a proof of 
concept for the use of FDGN 2 for both SEM and TEM, thus extending the multi-imaging capabilities of CLEM. This new method for multimodal CLEM imaging should enhance functional and structural analyses of biological organisms [4].

\section{References:}

[1] Uryu et al, Microscopy and Microanalysis 22(S3) (2017), p. 1142.

[2] Hoshino et al, Nature 527(7578) (2015), p. 329.

[3] Jones et al, Nat Biotechnol 28(2) (2010), p. 167.

[4] The current study was supported by The Rockefeller University (K.U., C.R.), Children's Cancer and Blood Foundation (D.L.) and Susan G. Komen Postdoctoral Fellowship (A.H.).
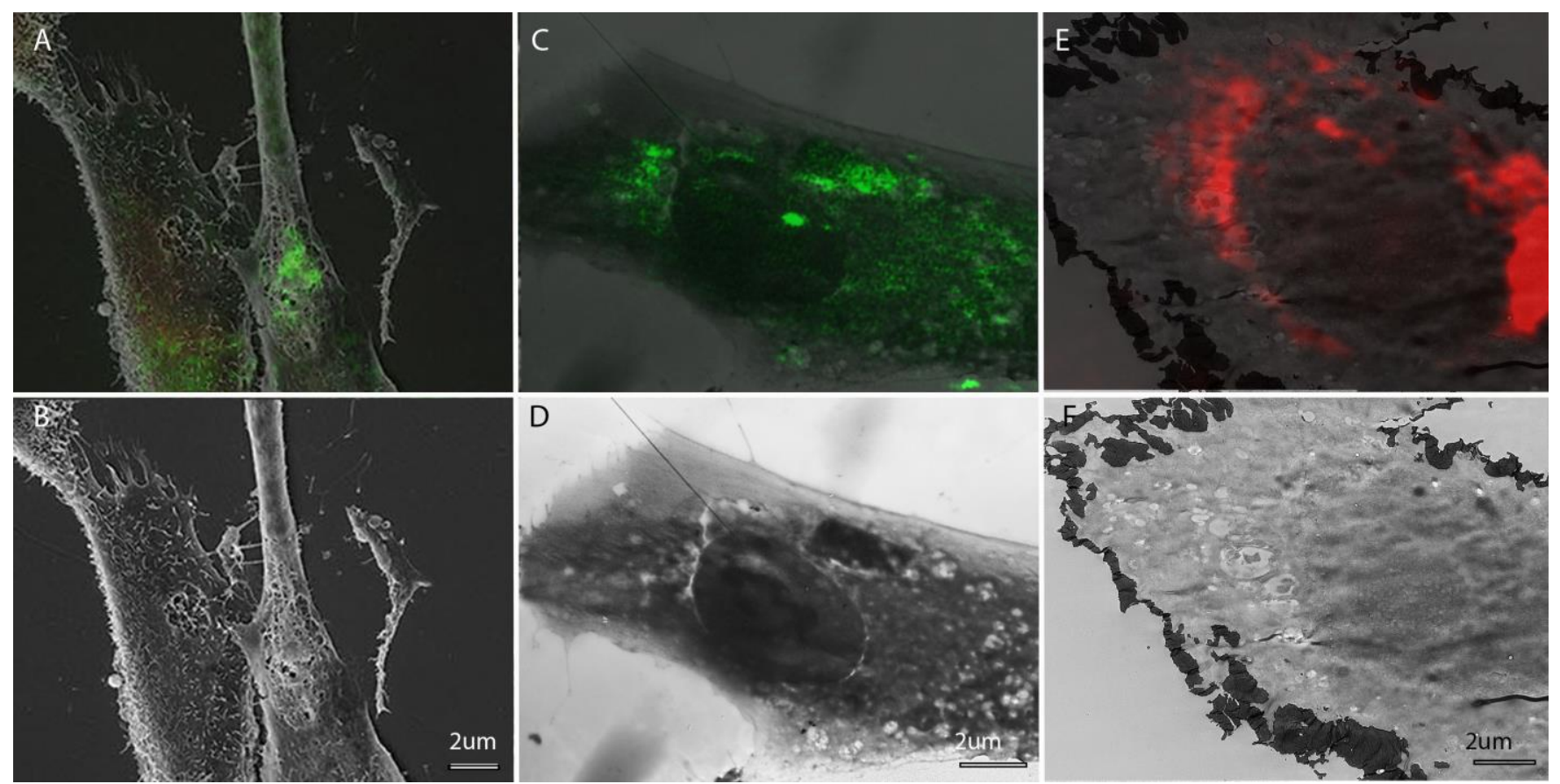

Figure 1. (A) Overlay of a fluorescent image taken with DeltaVision and a topographic view by SEM (Zeiss Leo1550) of a fibroblast (human primary lung fibroblasts WI-38) incubated with FM1-43(green) /PKA26 (red)-labelled exosomes ${ }^{2}$ and (B) its topographic view. (C) Combination of a fluorescence microgram by a wide-field epifluorescence microscope (Nikon Eclipse) and an electron microgram from a TEM thin section (JEOL 100CX) of fibroblast after the uptake of exogenous exosomes (labeled by FM1-43-green) $)^{2}$ and (D) its ultrastructure with a TEM thin section. (E) Combination of a fluorescence microgram by a wide-field epifluorescence microscope (labelled by FM1-43-green with Nikon Eclipse) and an electron microgram from a TEM thin section (JEOL 100CX) of a hepatoma cell (Huh-7.5) with RFP tagged mitochondrially-tethered IFN- $\beta$ promoter stimulator protein ${ }^{3}$ and $(\mathbf{F})$ its ultrastructure from a TEM thin section. Dark outline indicates a remaining conductive iridium coating used for SEM imaging prior to TEM sample preparation. In this case, the RFP signal was imaged prior to the conversion of the SEM sample to a TEM sample. 